\title{
The subgiant branch of $\omega$ Centauri seen through high-resolution spectroscopy
}

\section{The most metal-rich population $\star, \star \star$}

\author{
E. Pancino ${ }^{1}$, A. Mucciarelli ${ }^{2}$, P. Bonifacio ${ }^{3}$, L. Monaco ${ }^{4}$, and L. Sbordone ${ }^{3,5}$ \\ 1 INAF-Osservatorio Astronomico di Bologna, via Ranzani 1, 40127 Bologna, Italy \\ e-mail: elena.pancino@oabo.inaf.it \\ 2 Dipartimento di Astronomia, Università degli studi di Bologna, via Ranzani 1, 40127 Bologna, Italy \\ 3 GEPI, Observatoire de Paris, CNRS, Univ. Paris Diderot, 5 Place Jules Janssen, 92195 Meudon, France \\ ${ }^{4}$ European Southern Observatory, Casilla 19100, Santiago, Chile \\ 5 Max Planck Institute for Astrophysics, Karl-Schwarzschild-Str. 1, 85741 Garching, Germany
}

Received 30 May 2011 / Accepted 29 August 2011

\section{ABSTRACT}

\begin{abstract}
We analyze spectra of 18 stars belonging to the faintest subgiant branch in $\omega$ Centauri (the SGB-a), obtained with GIRAFFE@VLT at a resolution of $R \simeq 17000$ and a $S / N$ ratio between 25 and 50. We measure abundances of $\mathrm{Al}, \mathrm{Ba}, \mathrm{Ca}, \mathrm{Fe}, \mathrm{Ni}, \mathrm{Si}$, and $\mathrm{Ti}$ and we find that these stars have $\langle[\mathrm{Fe} / \mathrm{H}]\rangle=-0.73 \pm 0.14 \mathrm{dex}$, similarly to the corresponding red giant branch population (the RGB-a). We also measure $\langle[\alpha / \mathrm{Fe}]\rangle=+0.40 \pm 0.16 \mathrm{dex}$, and $\langle[\mathrm{Ba} / \mathrm{Fe}]\rangle=+0.87 \pm 0.23$ dex, in general agreement with past studies. It is very interesting to note that we found a uniform $\mathrm{Al}$ abundance, $\langle[\mathrm{Al} / \mathrm{Fe}]\rangle=+0.32 \pm 0.14$ dex, for all the 18 SGB-a stars analysed here, thus supporting past evidence that the usual (anti-)correlations are not present in this population, and suggesting a non globular cluster-like origin of this particular population. In the dwarf galaxy hypothesis for the formation of $\omega$ Cen, this population might be the best candidate for the field population of its putative parent galaxy, although some of its properties appear contradictory. It has also been suggested that the most metal-rich population in $\omega$ Cen is significantly enriched in helium. If this is true, the traditional abundance analysis techniques, based on model atmospheres with normal helium content, might lead to errors. We have computed helium enhanced atmospheres for three stars in our sample and verified that the abundance errors due to the use of non-enhanced atmospheres are negligible. Additional, indirect support to the enhanced helium content of the SGB-a population comes from our Li upper limits.
\end{abstract}

Key words. stars: abundances - globular clusters: individual: $\omega$ Centauri

\section{Introduction}

The region around the main sequence (hereafter MS) turn off (TO) and the subgiant branch (SGB) is the most sensitive of the colour-magnitude diagram (CMD) to age, and as such it has been the target of several studies to disentangle the agemetallicity relation in the complex mix of stellar populations of $\omega$ Centauri. The first studies employed photometric metallicity indicators (Hilker \& Richtler 2000; Hughes \& Wallerstein 2000), soon followed by low-resolution spectroscopic samples (Hughes et al. 2004; Hilker et al. 2004; Rey et al. 2004; Sollima et al. 2005b; Stanford et al. 2006; Villanova et al. 2007), coupled with exquisite new photometric catalogues (Ferraro et al. 2004; Bedin et al. 2004; Bellini et al. 2010), but the puzzle got deeper,

\footnotetext{
* Based on observations with the ESO GIRAFFE@VLT, under programme 079.D-0021(A). Also based on literature data obtained with WFI@VLT (programmes 62.L-0345 and 63.L-0439) and FORS@VLT (programme 68.D-0332). The following online databases were also extensively employed: NIST, VALD, Kurucz.

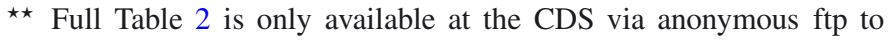
cdsarc.u-strasbg.fr $(130.79 .128 .5)$ or via

http://cdsarc.u-strasbg.fr/viz-bin/qcat?]/A+A/534/A53
}

as extensively discussed in the cited papers, and in the first paper of this series (Pancino et al. 2011, hereafter Paper I).

We focus here on the SGB-a, a sub-structure of the SGB of $\omega$ Cen, first found by Ferraro et al. (2004), and also named branch D by Villanova et al. (2007). The SGB-a appears to have a fainter magnitude than all other SGB components, and merges into the MS of $\omega$ Cen at a fainter magnitude than the TO of all other populations. There is nowadays no doubt left that the SGB-a is photometrically the same population as the RGB-a, the reddest and most metal-rich component of the red giant branch (RGB) of $\omega$ Cen (Pancino et al. 2000, 2002), as clearly visible in most recent high-quality photometries (see, e.g. Bedin et al. 2004; Villanova et al. 2007; Bellini et al. 2010). However, there is still some residual debate about its metallicity. High-resolution spectroscopic studies of RGB stars always find a metal-rich component with $[\mathrm{Fe} / \mathrm{H}]$ higher than $-1.0 \mathrm{dex}$, and generally around -0.6 or -0.7 dex on average (Pancino et al. 2002; Pancino 2003, 2004; Johnson \& Pilachowski 2010; Marino et al. 2011). Only two studies were performed on SGB-a stars, both based on lowresolution spectroscopy $(R \simeq 6000)$, and they give quite discrepant results. The former (Sollima et al. 2005b), based on calcium triplet measurements, found $[\mathrm{Fe} / \mathrm{H}] \simeq-0.6 \mathrm{dex}$, while the latter (Villanova et al. 2007) found $[\mathrm{Fe} / \mathrm{H}] \simeq-1.1 \mathrm{dex}$, with no 
star with $[\mathrm{Fe} / \mathrm{H}]$ above -1.0 dex in their sample, even if a handful of their targets belonged to the SGB-a, or branch $\mathrm{D}^{1}$.

Besides the problem of the SGB-a metallicity, there is also some doubt about the $\alpha$-elements enhancement of this metalrich population. Two studies found that RGB-a stars had lower $[\alpha / \mathrm{Fe}]$ with respect to MInt and MP stars (metal-intermediate and metal-poor, respectively, following the definition by Pancino et al. 2000), namely Pancino et al. (2002) and Origlia et al. (2003), the former containing three RGB-a stars studied with UVES and the latter more stars, but with SOFI low-resolution infrared spectroscopy. The confirmation of such a finding would be extremely important, because it would imply - in the selfenrichment framework for the formation of $\omega$ Cen - that type Ia supernovae had a chance to contribute to the chemical enrichment of this population. Unfortunately, more recent results cast some doubt on this result, or at least on its quantitative evaluation. In particular, Johnson \& Pilachowski (2010) found that a few stars in common with Pancino et al. (2002) had a higher $[\alpha / \mathrm{Fe}]$ than previously thought. It is clear, however, that a peculiar trend is visible in the behaviour of a few $\alpha$-elements, and in particular in [Ca/Fe] (see e.g., Norris \& Da Costa 1995; Smith 2004; Pancino 2004), where the enhancement is higher for RGB-MInt stars than for RGB-MP and RGB-a stars. In other words, $[\mathrm{Ca} / \mathrm{Fe}]$ rises slightly with metallicity, reaches its maximum value around $[\mathrm{Fe} / \mathrm{H}] \simeq-1.2 \mathrm{dex}$, and then gently decreases again. The amount of this variation is small, around $0.1 \mathrm{dex}$, and certainly below 0.2 dex, but it is clearly visible in the highresolution study by Johnson \& Pilachowski (2010), as well. Such a trend, if confirmed, is not straightforward to interpret - having most probably to do with a variable star formation rate and it requires a full modelization of the chemical evolution of $\omega$ Cen. In fact, while the intervention of type Ia supernovae could still be the best way to explain the (slight) decrease in $[\alpha / \mathrm{Fe}]$ of the RGB-a population, it is still difficult to understand why the MP population (metal-poor, with $[\mathrm{Fe} / \mathrm{H}] \simeq-1.7 \mathrm{dex}$ ) happens to have lower $[\alpha / \mathrm{Fe}]$ as well.

Finally, although no direct He measurement for the RGB-a exists (In Dupree et al. 2011, only stars up to the intermediate population were analyzed), it has been suggested by various authors (Norris 2004; Sollima et al. 2005b; Piotto et al. 2005; Renzini 2008) that the most metal-rich population in $\omega$ Cen should possess an enhanced helium content (ranging from $Y=0.35$ to $Y=0.40$, depending on the author). While this is understandable from the stellar evolution and nucleosynthesis point of view (Romano et al. 2010) ${ }^{2}$, if true it would pose a problem when one attempts to determine abundances from spectra using atmospheric models with a normal helium content. In Paper I, which dealt mainly with stars having $[\mathrm{Fe} / \mathrm{H}]<-1.0$ dex and $Y<0.30$, we provided an approximated estimate of the impact of helium on abundance calculations and found it irrelevant. When it comes to the SGB-a, however, a deeper analysis is required.

In this paper we analyse a sample of high-resolution spectra of SGB-a stars, selected from different photometry catalogues

\footnotetext{
1 A possible call to caution in this respect comes from Bellini et al. (2010), who found that the SGB-a could be split into two very close branches (see their Figs. 7, 9-11). Therefore, the properties of this population could be more complicated than expected.

2 In all the proposed self-enrichment scenarios, the most metal-rich population should also be the youngest (but see Sollima et al. 2005b, where it appears roughly coeval to the other populations), formed from gas already enriched in helium. Thus, in these scenarios, the RGB-a/SGB-a population should have at least the same helium abundance as the intermediate populations.
}

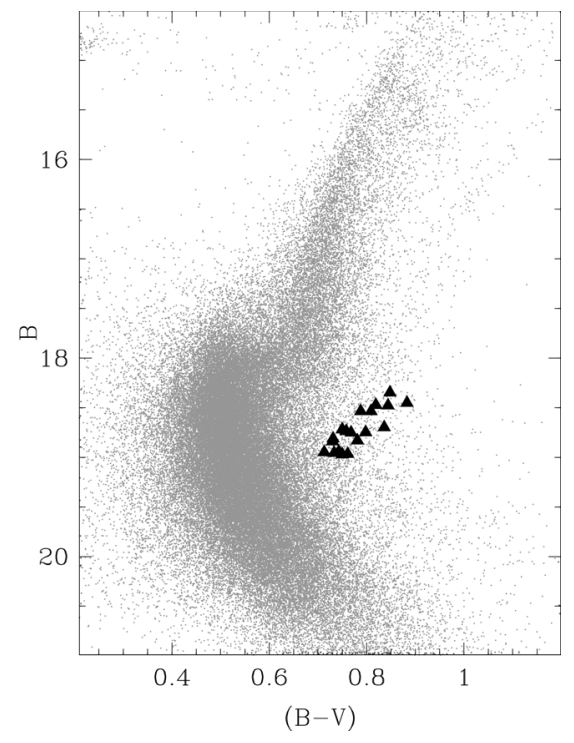

Fig. 1. Selected SGB-a stars (black triangles) on the FORS photometry (grey dots) by Sollima et al. (2005a).

(Pancino et al. 2000; Pancino 2003; Sollima et al. 2005a; Villanova et al. 2007) to represent the SGB-a component. The plan of the paper is the following: the data are presented in Sect. 2; the details of the abundance analysis are described in Sect. 3; the abundance results are discussed in Sect. 4; and in Sect. 5 we summarize our results and draw our conclusions.

\section{Observations and data reduction}

Observations were carried out betwen 27 and 29 April 2007, and the full dataset and data treatment are decribed in detail by Monaco et al. (2010). Among their data, we selected only stars clearly belonging to the SGB-a (as defined by Ferraro et al. 2004, and showed in Fig. 1) in all available photometries. The final list of targets, together with some basic information (see also Sect. 3.2) is reported in Table 1. The spectra were observed with GIRAFFE@VLT, using the HR15n setup (6430-6810 ̊), because the main goal of the original observations was the measurement of the lithium line at $6708 \AA$. The resolution was $R \simeq 17000$, the $S / N$ varied between 25 and 50 (see Table 1 ). Most our target were observed in one single exposure lasting $2 \mathrm{~h}$, while some of them were observed 9 times with a total exposure time of $17.3 \mathrm{~h}$ (see Table 1).

In short, the data were reduced with the 2.13 version of the GIRAFFE data reduction pipeline ${ }^{3}$. Seventeen fibres were allocated to sky observations on each plate, and the average of the closest fibers was subtracted from each target spectrum. After correcting for radial velocity differences with the IRAF ${ }^{4}$ task $f x$ cor, multiple spectra of each target were averaged together. A few stars which significantly deviated in their heliocentric velocity (derived with the IRAF task rvcorrect) from the cluster average $\left(V_{r}=232.8\right.$ or $233.4 \mathrm{~km} \mathrm{~s}^{-1}$, determined by Meylan et al. 1995; Pancino et al. 2007, respectively) were rejected. The final number of spectra surviving selection was 18 .

\footnotetext{
3 http://girbldrs. sourceforge.net/

4 http://iraf.noao.edu/. IRAF is distributed by the National Optical Astonomy Observatories, which is operated by the association of Universities for Research in Astronomy, Inc., under contract with the National Science Foundation.
} 
Table 1. Observing logs and atmospheric parameters.

\begin{tabular}{|c|c|c|c|c|c|c|c|c|c|c|c|}
\hline $\mathrm{ID}_{\mathrm{WFI}}{ }^{a}$ & $\mathrm{ID}_{\mathrm{FORS}}{ }^{b}$ & $\begin{array}{l}\text { RA (J2000) } \\
\quad(\mathrm{deg})\end{array}$ & $\begin{array}{c}\operatorname{Dec}(\mathrm{J} 2000) \\
(\mathrm{deg})\end{array}$ & $\begin{array}{c}V^{b} \\
(\mathrm{mag})\end{array}$ & $\begin{array}{c}(B-V)_{0}^{b} \\
(\mathrm{mag})\end{array}$ & $\begin{array}{c}\left(V-I_{J}\right)_{0}{ }^{b} \\
(\mathrm{mag})\end{array}$ & $n_{\mathrm{obs}}$ & $S / N$ & $\begin{array}{l}T_{\mathrm{eff}}^{H_{\alpha}} \\
(\mathrm{K})\end{array}$ & $\begin{array}{l}\log g \\
(\operatorname{dex})\end{array}$ & $\begin{array}{c}v_{\mathrm{t}} \\
\left(\mathrm{km} \mathrm{s}^{-1}\right)\end{array}$ \\
\hline 213129 & 33807 & 201.7662757 & -47.5488277 & 17.73 & 0.70 & 0.99 & 1 & 35 & 5300 & 3.8 & 1.0 \\
\hline 213295 & 40145 & 201.6777863 & -47.5484132 & 17.98 & 0.66 & 0.91 & 9 & 45 & 5500 & 3.9 & 1.0 \\
\hline 214198 & 40987 & 201.6423287 & -47.5449255 & 18.22 & 0.62 & 0.88 & 1 & 26 & 5450 & 4.0 & 1.0 \\
\hline 215315 & 65607 & 201.7166701 & -47.5407130 & 17.75 & 0.68 & 0.85 & 1 & 35 & 5650 & 3.9 & 1.0 \\
\hline 215700 & 37198 & 201.6256851 & -47.5393905 & 18.11 & 0.62 & 0.89 & 9 & 49 & 5500 & 4.0 & 1.0 \\
\hline 215931 & 61617 & 201.7105924 & -47.5383614 & 18.05 & 0.67 & 0.86 & 1 & 37 & 5650 & 4.0 & 1.0 \\
\hline 216031 & 34467 & 201.6134380 & -47.5381179 & 17.65 & 0.71 & 1.03 & 9 & 49 & 5250 & 3.7 & 1.0 \\
\hline 218364 & 29483 & 201.7772506 & -47.5289457 & 17.50 & 0.74 & 0.98 & 1 & 33 & 5300 & 3.7 & 1.0 \\
\hline 220401 & 42942 & 201.6264389 & -47.5217612 & 18.22 & 0.64 & 0.99 & 1 & 25 & 5250 & 3.9 & 1.0 \\
\hline 220947 & 29233 & 201.7634185 & -47.5195723 & 17.57 & 0.77 & 1.03 & 1 & 37 & 5500 & 3.8 & 1.0 \\
\hline 224701 & 63733 & 201.7160750 & -47.5054148 & 17.86 & 0.73 & 0.89 & 1 & 38 & 5500 & 3.9 & 1.0 \\
\hline 224921 & 33448 & 201.7852963 & -47.5044375 & 17.98 & 0.65 & 0.96 & 9 & 47 & 5500 & 3.9 & 1.0 \\
\hline 227902 & 30356 & 201.7883906 & -47.4929578 & 18.21 & 0.65 & 0.61 & 1 & 32 & 5750 & 4.1 & 1.0 \\
\hline 234254 & 33737 & 201.6024956 & -47.4688537 & 17.97 & 0.64 & 0.99 & 1 & 30 & 5350 & 3.9 & 1.0 \\
\hline 235569 & 62919 & 201.6354387 & -47.4637003 & 17.95 & 0.69 & 0.98 & 1 & 39 & 5650 & 4.0 & 1.0 \\
\hline 243327 & 37860 & 201.6368456 & -47.4344181 & 17.63 & 0.73 & 1.04 & 9 & 48 & 5300 & 3.7 & 1.0 \\
\hline 247798 & 37337 & 201.6311023 & -47.4175838 & 18.24 & 0.60 & 0.96 & 9 & 45 & 5500 & 4.0 & 1.0 \\
\hline 248814 & 38329 & 201.7716525 & -47.4135293 & 18.08 & 0.62 & 0.89 & 9 & 43 & 5500 & 4.0 & 1.0 \\
\hline
\end{tabular}

Notes. ${ }^{(a)}$ Photometry from Pancino et al. (2000). ${ }^{(b)}$ Photometry from Sollima et al. (2005a).

\section{Abundance analysis}

\subsection{Equivalent widths and atomic data}

We selected the majority of our lines and their atomic data from the VALD ${ }^{5}$ database (Kupka et al. 1999). The NIST $^{6}$ atomic data were employed for spectral syntesis of the Ba II line at $6496 \AA$, including hyperfine structure (HFS) and isotope splitting. To identify reliable lines, the unfiltered linelist of Paper I was compared to the spectral range of the GIRAFFE spectra, and only reliable, unblended, and relatively strong lines were retained. DAOSPEC ${ }^{7}$ (Stetson \& Pancino 2008) was used to measure equivalent widths $(E W)$ of all the chosen lines. A first pass abundance analysis was performed (Sect. 3.3): lines that showed systematically higher errors and bad Q parameters (see Stetson \& Pancino 2008; Pancino et al. 2010, for details) and that simultaneously gave systematically discrepant abundances were rejected. Finally, all lines that had $E W<15 \mathrm{~m} \AA$, or $E W>150 \mathrm{~m} \AA$ were not used to determine abundances. The DAOSPEC $E W$ measurements used for the abundance analysis are shown in Table 2, along with the formal error $\delta E W$ and the quality parameter $Q$ for each line (see Stetson \& Pancino 2008, for details).

To check our DAOSPEC measurements, we re-measured the $E W$ of six stars (Fig. 2) with the IRAF task splot. The average EW difference, computed on a total of 218 lines, was $-1.8 \pm 9.7 \mathrm{~m} \AA$ for the three highest $S / N$ stars and $-4.2 \pm 14.6 \mathrm{~m} \AA$ for the three lowest $S / N$ stars. All differences indicate that the IRAF measurements were slightly lower than the DAOSPEC ones, but the two sets of EW are totally compatible within the uncertainties.

\footnotetext{
5 http://www.astro.uu. se/ vald/

${ }^{6}$ http://physics.nist.gov/PhysRefData/ASD/index.html

7 Available at http://www.bo.astro.it/ pancino/projects/ daospec.html, http://www2.cadc-ccda.hia-iha.nrc-cnrc. gc.ca/community/STETSON/daospec/
}

Table 2. Equivalent widths and atomic data for the lines used in the classical abundance analysis of the program stars.

\begin{tabular}{cccccccc}
\hline \hline Star & $\begin{array}{c}\lambda \\
(\AA)\end{array}$ & $\mathrm{El}$ & $\begin{array}{c}\chi_{\mathrm{ex}} \\
(\mathrm{eV})\end{array}$ & $\begin{array}{c}\log g f \\
(\mathrm{dex})\end{array}$ & $\begin{array}{c}E W \\
(\mathrm{~m} \AA)\end{array}$ & $\begin{array}{c}\delta E W \\
(\mathrm{~m} \AA)\end{array}$ & $Q$ \\
\hline 213129 & 6499.65 & $\mathrm{CaI}$ & 2.52 & -0.72 & 94.7 & 0.09 & 0.915 \\
213129 & 6572.78 & $\mathrm{CaI}$ & 0.00 & -4.10 & 44.0 & 0.19 & 1.291 \\
213129 & 6717.68 & $\mathrm{CaI}$ & 2.71 & -0.60 & 95.6 & 0.08 & 0.532 \\
213129 & 6469.19 & $\mathrm{FeI}$ & 4.83 & -0.77 & 62.0 & 0.20 & 1.533 \\
213129 & 6475.62 & FeI & 2.56 & -2.94 & 91.7 & 0.14 & 1.459 \\
\hline
\end{tabular}

Notes. The complete version of the Table is available at CDS. Here we show a few lines to illustrate its contents.

\subsection{Atmospheric parameters}

The HR15n setup of GIRAFFE (643-681 nm, approximately) contains few lines for these subgiants, since it was mainly chosen to measure the $\mathrm{Li}$ abundance and to have a precise estimate of $T_{\text {eff }}$ (Monaco et al. 2010) using the $\mathrm{H}_{\alpha}$ line wings. Therefore, we could not perform a full spectroscopic analysis as in Paper I, and we adopted fixed atmospheric parameters for our stars.

For temperatures, we used two independent estimates. The former is based on the FORS photometry (Sollima et al. 2005a), where dereddened $(B-V)_{0}$ and $(V-I)_{0}$ colors were obtained from $B, V$ and $I$ magnitudes adopting $E(B-V)=0.11$ (Lub 2002) and $E(V-I) / E(B-V)=1.30$ (Dean et al. 1978), and are listed in Table 1 along with the $V$ magnitudes. The $V-I$ color was converted from the original $V-I_{C}$, based on the Cousins $I$ magnitude, to the $V-I_{J}$, based on the Johnsons $I$ magnitude, with the relations by Bessell (1979). Effective temperatures (hereafter $T_{\text {eff }}$ ) were then obtained with the Alonso et al. (1999) calibration. The latter independent method is based on the profile fitting of the $\mathrm{H}_{\alpha}$ line wings, employing a modified version of the BALMER ${ }^{8}$ code, which uses the self-broadening theory by Barklem et al. (2000) and the Stark broadening by

8 The original version provided by Kurucz can be found at http://kurucz.harvard. edu/. 


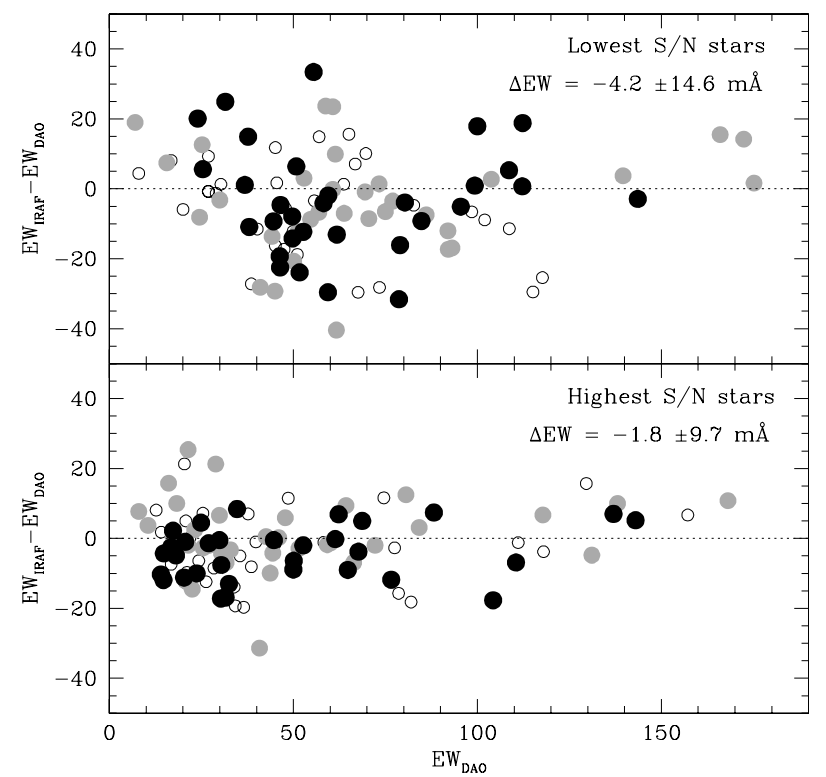

Fig. 2. Comparison between DAOSPEC and IRAF measurements for all elements. The top panel shows the three highest $S / N$ stars WFI 215700 (black points), WFI 224921 (white points), and WFI 248814 (grey points). The bottom panel shows the three lowest $S / N$ stars WFI 214198 (black points), WFI 220401 (grey points), and WFI 234254 (white points). Perfect agreement is marked with a dotted line in both panels, along with the average differences and sigmas.

Stehlé \& Hutcheon (1999). A comparison between the resulting $T_{\text {eff }}$ values is shown in Fig. 3. The average difference between photometric $(B-V)$ temperatures and spectroscopic $\mathrm{H}_{\alpha}$ ones is $+34 \pm 174 \mathrm{~K}$, while the one between the $(V-I)$ and $\mathrm{H}_{\alpha}$ temperatures is $61 \pm 119 \mathrm{~K}$. Given the good agreement, we arbitrarily ${ }^{9} \mathrm{de}-$ cided to adopt the $\mathrm{H}_{\alpha}$ temperatures for our analysis (Table 1) ${ }^{10}$, with an estimated uncertainty of the order of $\pm 100 \mathrm{~K}$.

Gravities were derived with the adopted $T_{\text {eff }}$ values, the $\mathrm{BC}_{V}$ from Alonso et al. (1999) as mentioned above, and a distance modulus $(m-M)_{V}=14.04 \pm 0.11 \mathrm{mag}$ (Bellazzini et al. 2004), by means of fundamental relations:

$\log g_{*}=0.4\left(M_{V}+B C_{V}\right)+4 \log T_{\mathrm{eff}, *}-12.61$

where the solar values where assumed in conformity with the IAU recommendations (Andersen 1999), i.e., $\log g_{\odot}=4.437$, $T_{\mathrm{eff}, \odot}=5770 \mathrm{~K}$ and $M_{\mathrm{bol}, \odot}=4.75$. A typical mass of $0.8 M_{\odot}$ was assumed for the program stars (Bergbusch \& VandenBerg 2001). The final $\log g$ values are listed in Table 1 , and we estimated an uncertainty of \pm 0.2 dex.

Finally, we verified that the microturbulent velocity, $v_{\mathrm{t}}$, is poorly constrained by the limited number of Fe lines at our disposal, so we adopted $v_{\mathrm{t}}=1.0 \mathrm{~km} \mathrm{~s}^{-1}$ for all our SGB-a targets (see Marino et al. 2008, as well), as was also done by Monaco et al. (2010), and we allowed for a conservative uncertainty of $\pm 0.3 \mathrm{~km} \mathrm{~s}^{-1}$.

\footnotetext{
${ }_{9}$ Even if the trend is probably not significant, it can be seen in Fig. 3 that temperatures derived from the $(B-V)$ colour do not show a flat difference with temperatures determined with $\mathrm{H}_{\alpha}$ or $(V-I)$. This could be due to a small residual colour term in the the $B$ magnitude calibration by Sollima et al. (2005a).

${ }_{10}$ The numbers in Table 1 differ slightly from those in Table 2 by Monaco et al. (2010), because here we used synthetic spectra built with atmosphere models of -1.0 dex in metallicity, while there they showed values derived assuming -1.5 dex.
}

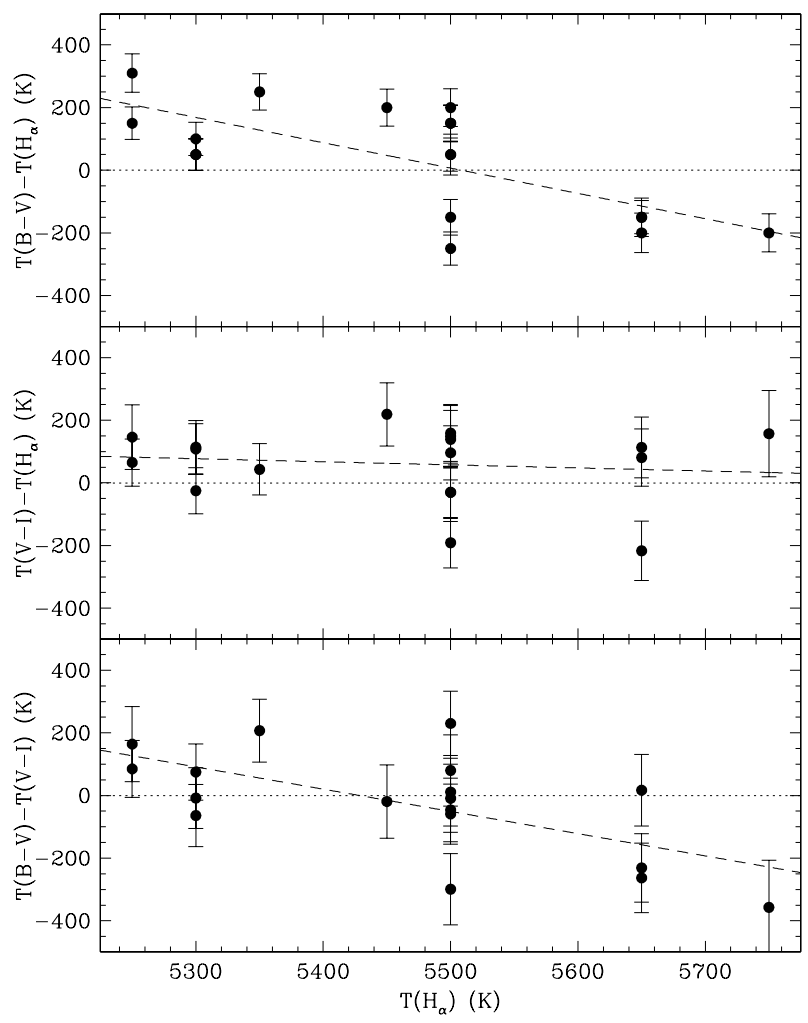

Fig. 3. Comparison between photometric temperatures based on the $B-$ $V$ and $V-I$ colours and the spectroscopic ones based on the $\mathrm{H}_{\alpha}$ profile fitting (see text).

\subsection{Abundance calculations}

For all chemical species except Ba (see Sect. 4.4), we computed abundances with the help of the updated version of the original code by Spite (1967). Our reference solar abundance was Grevesse et al. (1996). We used the new plane-parallel MARCS $^{11}$ model atmospheres with standard composition ${ }^{12}$. We chose the closest available global model metallicity (taking into account $\alpha$-enhancement) to the $\omega$ Cen sub-populations, which was -1.0 dex for all targets.

For all species we computed a $3 \sigma$-clipped average of abundances resulting from each available line. For Fe and $\mathrm{Ti}$, which had both neutral and ionized lines, we computed the weighted (on the number of lines) average of the two ionization stages to obtain $[\mathrm{Fe} / \mathrm{H}]$ and $[\mathrm{Ti} / \mathrm{Fe}]^{13}$. We typically rejected lines that had $E W>150 \mathrm{~m} \AA$, where the Gaussian approximation could fail, or $E W<15 \mathrm{~m} \AA$, since the relative error was too high. For $\mathrm{Ca}$, one line at $6462 \AA$ was larger than $150 \mathrm{~m} \AA$, but we checked that the DAOSPEC measurement were not too underestimated by visually inspecting the spectrum and overlaying the DAOSPEC Gaussian fit on each target star.

\footnotetext{
${ }^{11}$ http://marcs.astro.uu.se/

12 This means $[\alpha / \mathrm{Fe}]=+0.4$ for metal-poor stars of $[\mathrm{Fe} / \mathrm{H}]<-1.0$ and reaching $[\alpha / \mathrm{Fe}]=0$ at $[\mathrm{Fe} / \mathrm{H}]=0$, following schematically the typical halo-disk behaviour of the Milky Way field population.

13 The abundance of the few Ti I and II lines were so scattered that the results presented in Sect. 4.3 would not change significantly if we used a straight average.
} 
E. Pancino et al.: The most metal-rich population in $\omega$ Centauri. II.

Table 3. Abundance ratios with random uncertainties (see text).

\begin{tabular}{|c|c|c|c|c|c|c|c|c|}
\hline $\mathrm{ID}_{\mathrm{WFI}}{ }^{a}$ & $\mathrm{ID}_{\mathrm{FORS}}^{b}$ & $\begin{array}{c}{[\mathrm{Fe} / \mathrm{H}]} \\
(\mathrm{dex})\end{array}$ & $\begin{array}{c}{[\mathrm{Al} / \mathrm{Fe}]} \\
(\mathrm{dex})\end{array}$ & $\begin{array}{c}{[\mathrm{Ba} / \mathrm{Fe}]} \\
(\mathrm{dex})\end{array}$ & $\begin{array}{c}{[\mathrm{Ca} / \mathrm{Fe}]} \\
(\mathrm{dex})\end{array}$ & $\begin{array}{c}{[\mathrm{Ni} / \mathrm{Fe}]} \\
(\mathrm{dex})\end{array}$ & $\begin{array}{c}{[\mathrm{Si} / \mathrm{Fe}]} \\
(\mathrm{dex})\end{array}$ & $\begin{array}{c}{[\mathrm{Ti} / \mathrm{Fe}]} \\
(\mathrm{dex})\end{array}$ \\
\hline 13129 & 33807 & $-0.45 \pm 0.14$ & $+0.39 \pm 0.17$ & $0.82 \pm \ldots$ & $+0.18 \pm 0.16$ & $-0.14 \pm 0.28$ & $+0.47 \pm \ldots$ & $+0.29 \pm 0.54$ \\
\hline & & & & & & & & \\
\hline & & & & & & & & \\
\hline & & & & & & & & \\
\hline & & & & & & & & \\
\hline & & & & & & & $\pm \ldots$ & \\
\hline & & & & & & & $\pm \ldots$ & \\
\hline & & & & & & & $\pm \ldots$ & \\
\hline & 942 & & & $17 \pm \ldots$ & & & $+0.88 \pm \ldots$ & .55 \\
\hline & 233 & 9 & 14 & $0.90 \pm \ldots$ & +0 . & & $+0.33 \pm \ldots$ & 0.35 \\
\hline & & & &.$\pm \ldots$ & +0 . & & $1 \pm \ldots$ & 0.61 \\
\hline & & & & $1.18 \pm$. & & & $\pm \ldots$ & 0.47 \\
\hline & & & $+0.47 \pm 0.19$ & $\ldots \pm \ldots$ & $+0.02 \pm$ & $-0.27 \pm$ & $+0.58 \pm \ldots$ & +0.6 \\
\hline 234254 & 33737 & $-0.76 \pm 0.14$ & $+0.26 \pm \ldots$ & $0.51 \pm \ldots$ & $+0.24 \pm$ & $+0.11 \pm$ & $+0.98 \pm \ldots$ & $+0.59 \pm 0.51$ \\
\hline & 2919 & & $\ldots \quad \pm$ & $\ldots \pm \ldots$ & $-0.08 \pm 0.27$ & $-0.08 \pm 0$ & $+0.52 \pm \ldots$ & $+0.61 \pm 0.66$ \\
\hline & & & $+0.29 \pm 0.09$ & $0.99 \pm \ldots$ & $+0.47 \pm 0.11$ & $-0.08 \pm 0.13$ & $+0.70 \pm \ldots$ & $+0.16 \pm 0.34$ \\
\hline & & & & $0.97 \pm \ldots$ & $+0.51 \pm 0.11$ & & $+0.55 \pm \ldots$ & $+0.57 \pm 0.35$ \\
\hline 248814 & 38329 & $-0.81 \pm 0.08$ & $+0.48 \pm 0.19$ & $1.03 \pm \ldots$ & $+0.37 \pm 0.10$ & $-0.03 \pm 0.13$ & $+0.61 \pm \ldots$ & $+0.38 \pm 0.37$ \\
\hline
\end{tabular}

Table 4. Uncertainties due to the choice of stellar parameters.

\begin{tabular}{|c|c|c|c|c|c|c|c|}
\hline \multirow[b]{2}{*}{ El } & \multicolumn{3}{|c|}{ Star WFI 216031} & \multicolumn{3}{|c|}{ Star WFI 227902} & \multirow{2}{*}{$\begin{array}{c}\text { Average } \\
\Delta[\mathrm{El} / \mathrm{Fe}] \\
\text { (dex) }\end{array}$} \\
\hline & $\begin{array}{c} \pm 100 \mathrm{~K} \\
(\mathrm{dex})\end{array}$ & $\begin{array}{c} \pm 0.3 \mathrm{~km} \mathrm{~s}^{-1} \\
(\mathrm{dex})\end{array}$ & $\begin{array}{l}\text { Total } \\
(\mathrm{dex})\end{array}$ & $\begin{array}{c} \pm 100 \mathrm{~K} \\
(\mathrm{dex})\end{array}$ & $\begin{array}{c} \pm 0.3 \mathrm{~km} \mathrm{~s}^{-1} \\
\quad(\mathrm{dex})\end{array}$ & $\begin{array}{l}\text { Total } \\
\text { (dex) }\end{array}$ & \\
\hline$\delta[\mathrm{Fe} / \mathrm{H}]$ & \pm 0.06 & \pm 0.02 & \pm 0.06 & \pm 0.05 & \pm 0.02 & \pm 0.05 & \pm 0.06 \\
\hline$\delta[\mathrm{Al} / \mathrm{Fe}]$ & \pm 0.05 & \pm 0.12 & \pm 0.13 & \pm 0.05 & \pm 0.13 & \pm 0.14 & \pm 0.14 \\
\hline$\delta[\mathrm{Ba} / \mathrm{Fe}]$ & \pm 0.09 & \pm 0.06 & \pm 0.11 & \pm 0.37 & \pm 0.37 & \pm 0.52 & \pm 0.32 \\
\hline$\delta[\mathrm{Ca} / \mathrm{Fe}]$ & \pm 0.10 & \pm 0.08 & \pm 0.13 & \pm 0.09 & \pm 0.05 & \pm 0.10 & \pm 0.12 \\
\hline$\delta[\mathrm{Ni} / \mathrm{Fe}]$ & \pm 0.09 & \pm 0.09 & \pm 0.12 & \pm 0.09 & \pm 0.03 & \pm 0.09 & \pm 0.11 \\
\hline$\delta[\mathrm{Si} / \mathrm{Fe}]$ & \pm 0.00 & \pm 0.02 & \pm 0.02 & \pm 0.02 & \pm 0.02 & \pm 0.03 & \pm 0.03 \\
\hline$\delta[\mathrm{Ti} / \mathrm{Fe}]$ & \pm 0.07 & \pm 0.03 & \pm 0.08 & \pm 0.06 & \pm 0.05 & \pm 0.08 & \pm 0.08 \\
\hline
\end{tabular}

\subsection{Abundance uncertainties}

For those elements that had more than one line after $\sigma$-clipping, we estimated the random (internal) uncertainty as $\sigma / \sqrt{n}$, as reported in Table 3. When only one line was available, we put an ellipsis in Table 3, and a rough estimate of the associated random uncertainty is $\simeq 0.10 \mathrm{dex}$, according to the Cayrel (1988) formula.

Another source of uncertainty is the global accuracy of the continuum normalization. We used the r.m.s. of the residual spectrum calculated by DAOSPEC after removing all the fitted spectral lines, which was on average $\pm 4 \%$ for our GIRAFFE spectra. According to Fig. 2 by Stetson \& Pancino (2008), this corresponds roughly to a constant error of $\pm 10 \mathrm{~m} \AA$, which in turn corresponds to approximately $\pm 0.10 \mathrm{dex}$ in our abundances.

Finally, to estimate the uncertainty due to the choice of atmospheric parameters, we cannot use the Cayrel et al. (2004) method, since we did not determine our parameters with the classical spectroscopic optimization method. In our case, $T_{\text {eff }}$ and $v_{\mathrm{t}}$ are determined in a fully independent way, while $\log g$ is fixed by the choice of $T_{\text {eff }}$ (see Sect. 3.2). Therefore, we re-computed our abundances by altering separately $T_{\text {eff }}($ by $\pm 100 \mathrm{~K})$ and $v_{\mathrm{t}}$ (by $\pm 0.3 \mathrm{dex}$ ) for one of the coolest (WFI 216031) and of the warmest (WFI 227902) stars. For each of the $\pm 100 \mathrm{~K}$ models, we adopted the appropriate value of $\log g$ from Eq. (1). We computed therefore the uncertainties due to $T_{\text {eff }}$ as the average of the abundance variations for the $+100 \mathrm{~K}$ and the $-100 \mathrm{~K}$ models, and we did the same for $v_{\mathrm{t}}$. The two uncertainties were then summed in quadrature (since the two parameters are determined in a fully independent way) to yield an uncertainty for each of the two stars, and the average of the two stars was taken as our estimate of the parameters choice impact on our abundance ratios, as listed in Table 4. For those elements with two ionization stages ( $\mathrm{Ti}$ and $\mathrm{Fe}$ ), we computed the abundance ratio exactly as described in Sect. 3.3, i.e., using a weighted average on the two ionization stages.

\subsection{Literature comparison of star WFI 214198}

We found one star in common with Villanova et al. (2007), which is star WFI 214198 in the Pancino et al. (2000) WFI catalogue, corresponding to star 28448 in their Table 1. The atmospheric parameters agree well, because they found $T_{\text {eff }}=5400 \mathrm{~K}$, $\log g=4.1 \mathrm{dex}$, and $v_{\mathrm{t}}=1.0 \mathrm{~km} \mathrm{~s}^{-1}$. They used the Grevesse \& Sauval (1998) solar composition, which is practically identical to our Grevesse et al. (1996).

However, we found a significant difference in the $[\mathrm{Fe} / \mathrm{H}]$ ratio, because they measure $[\mathrm{Fe} / \mathrm{H}]=-1.12 \pm$ $0.08( \pm 0.15-0.20)$ dex, while we found $-0.55 \pm 0.16( \pm 0.06)$ dex, where the uncertanties in parenthesis are systematic, due to the uncertainty in stellar parameters. Concerning other elements, we found: $\Delta[\mathrm{Ca} / \mathrm{Fe}]=+0.20 \operatorname{dex}, \Delta[\mathrm{Ti} / \mathrm{Fe}]=-0.45 \mathrm{dex}$, $\Delta[\mathrm{Ba} / \mathrm{Fe}]=0.46 \mathrm{dex}$, where all differences are computed by subtracting our measurements from the Villanova et al. (2007) ones. If we compare the ratios with respect to hydrogen, we find: $\Delta[\mathrm{Ca} / \mathrm{H}]=-0.27 \operatorname{dex}, \Delta[\mathrm{Ti} / \mathrm{H}]=-0.92 \operatorname{dex}, \Delta[\mathrm{Ba} / \mathrm{H}]=$ -0.01 dex. Therefore, given the uncertainties of both studies, we 
can conclude that the calcium and barium ratios are compatible with each other, while the titanium discrepancy is most probably due to our uncertainties for this element being significantly higher than for other elements.

The discrepancy in the iron abundances requires instead a deeper discussion. Given that we used the same atmospheric parameters, similar solar composition, and atmospheric models with a similar thermal structure, we can only ascribe the discrepancy to the different spectral quality, because the Villanova et al. (2007) analysis is based on $R \simeq 6400$ spectra (ours have $R \simeq 17000$ ), although with higher $S / N \simeq 100-150$ (ours have $S / N \simeq 25-50$ ). In particular, for deriving their $[\mathrm{Fe} / \mathrm{H}]$ ratios, they analyzed a short and blue spectral region (4400-4425 $\AA)^{14}$, where metal lines blanketing is high, and highly uncertain (Kurucz 1992; Munari et al. 2005; Bertone et al. 2008). Therefore, the uncertainty in the continuum placement can become even more problematic than usual ${ }^{15}$, especially at low resolution. In this respect, we recall here that a different low resolution study of SGB-a stars, based on the infrared calcium triplet (Sollima et al. 2005b), found an average $[\mathrm{Fe} / \mathrm{H}] \simeq-0.6$ dex. On the other hand, the SGB-a (or branch D), clearly merges with the RGB-a in all published photometries (Bedin et al. 2004; Villanova et al. 2007; Bellini et al. 2010), and all high-resolution measurements of RGB-a stars provide abundances higher than $[\mathrm{Fe} / \mathrm{H}]>-1.0$ dex (see, e.g. Pancino et al. 2002; Pancino 2004; Johnson \& Pilachowski 2010; Marino et al. 2011). In conclusion, we are confident that our measurements are correct, within the quoted uncertainties.

\section{Abundance results}

Among the several literature studies which provide abundance ratios, we selected two to compare with our results, since they are the ones that contain the largest samples of metal-rich $([\mathrm{Fe} / \mathrm{H}]>-1.0 \mathrm{dex})$ stars. The former is the study of more than 800 red giants by Johnson \& Pilachowski (2010), which has similar resolution to the GIRAFFE spectra presented here, and agrees well with most previous studies (see their Fig. 6) so it serves as a good comparison dataset. The latter is the low resolution study by Villanova et al. (2007), which studies subgiant stars very similar to the ones analysed here (but see Sect. 3.5). In addition, we found a large sample of $[\mathrm{Ba} / \mathrm{Fe}]$ determinations of red giants at all metallicities in Marino et al. (2011), based on GIRAFFE spectra as well. These abundance ratios are plotted in Fig. 4 along with our measurements, and are discussed in the following Sections. While we corrected for the solar abundance reference values differences, and we tried to check $\log g f$ values when possible, it is clear that residual zeropoint dfferences might be present in the comparison.

\footnotetext{
14 Other elements were measured from a handful of lines in slightly different spectral regions, and that probably explains why their abundances are less different from ours.

15 Villanova et al. (2007) used full spectral synthesis of the spectral region between $4400-4425 \AA$ to derive $[\mathrm{Fe} / \mathrm{H}]$, thus the "overblanketing" predicted by theoretical $\log g f$ (from the Kurucz linelist) of tiny iron lines could in principle induce a continuum misplacement, causing an underestimate of the $[\mathrm{Fe} / \mathrm{H}]$ ratio, such as is evident in the comparison presented here. A quantitative analysis of the "overblanketing" effect is out of the scope of the present paper, but the effect goes in the right direction.
}

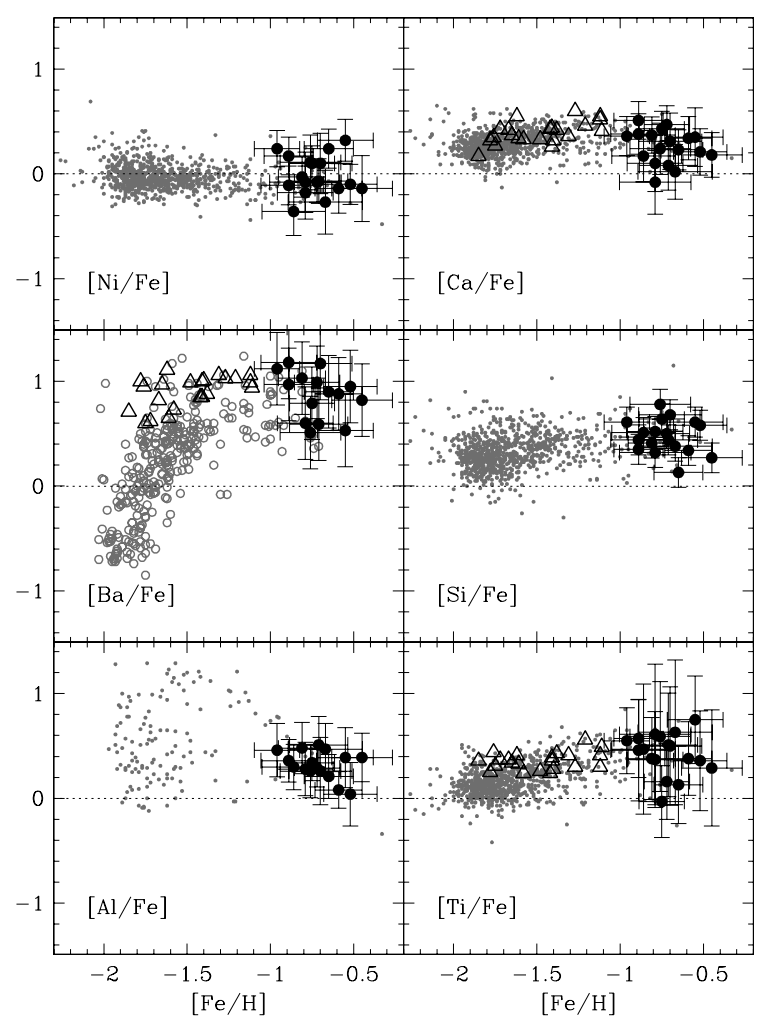

Fig. 4. Abundance ratios for all measured elements. In all panels, grey dots are the measurements by Johnson \& Pilachowski (2010), grey empty circles by Marino et al. (2011), the empty triangles the ones by Villanova et al. (2007), and the black dots with errorbars our measurements.

\subsection{Iron-peak elements}

We could measure $\sim 10-15$ Fe I lines, depending on the star, and only one Fe II line, which gave discordant abundances and was discarded. We found an average $\langle[\mathrm{Fe} / \mathrm{H}]\rangle=-0.73 \pm 0.14 \mathrm{dex}$. As discussed in Sect. 3.5, a significantly lower iron abundance was found by Villanova et al. (2007), based on low-resolution spectra, but our measurements agree very well with past abundance determinations of the RGB-a component (Pancino et al. 2002; Origlia et al. 2003; Pancino 2003, 2004; Johnson \& Pilachowski 2010; Marino et al. 2011), and with the SGB-a abundance determination by Sollima et al. (2005b).

We could also measure 3-4 Ni lines, depending on the star, and we found an average $\langle[\mathrm{Ni} / \mathrm{Fe}]\rangle=-0.02 \pm 0.19 \mathrm{dex}$, which agrees as well with the results by Johnson \& Pilachowski (2010). A solar ratio of $[\mathrm{Ni} / \mathrm{Fe}]$ is of course expected because $\mathrm{Ni}$ is produced in the same site as Fe.

\subsection{Lithium}

We derived lithium upper limits from the $6708 \AA \mathrm{Li}$ I line (Table 5) of all the 18 SGB-a stars presented in this paper ${ }^{16}$. We used the fitting formulas of Sbordone et al. (2010) for 1D LTE and 3D NLTE: the difference among the two is very small. The

16 The limits are derived with a different method, assumed metallicity and parameters than in Monaco et al. (2010), and this explains the small differences in the two sets of upper limits, for the 7 stars in common. The Sbordone et al. (2010) fitting formulas we used are based on 3D CO ${ }^{5}$ BOLD models (Freytag et al. 2002, 2010) and 1D LHD models, while Monaco et al. (2010) used ATLAS models. As can be seen, such differences are unimportant. 
Table 5. Upper limits on Li abundances, for $2 \sigma_{\mathrm{ew}}$.

\begin{tabular}{|c|c|c|c|c|}
\hline $\mathrm{ID}_{\mathrm{WFI}}$ & $\begin{array}{c}T_{\text {eff }} \\
\mathrm{K}\end{array}$ & $\begin{array}{l}\log g \\
\text { c.g.s. }\end{array}$ & $\begin{array}{c}A(\mathrm{Li}) \\
1 \mathrm{DTE}\end{array}$ & $\begin{array}{c}A(\mathrm{Li}) \\
\text { 3D NLTE }\end{array}$ \\
\hline 213129 & 5300 & 3.80 & $<1.31$ & $<1.23$ \\
\hline 213295 & 5500 & 3.90 & $<1.35$ & $<1.30$ \\
\hline 214198 & 5450 & 4.00 & $<1.55$ & $<1.49$ \\
\hline 215315 & 5650 & 3.90 & $<1.58$ & $<1.56$ \\
\hline 215700 & 5500 & 4.00 & $<1.30$ & $<1.25$ \\
\hline 215931 & 5650 & 4.00 & $<1.55$ & $<1.52$ \\
\hline 216031 & 5250 & 3.70 & $<1.12$ & $<1.04$ \\
\hline 218364 & 5300 & 3.70 & $<1.34$ & $<1.27$ \\
\hline 220401 & 5250 & 3.90 & $<1.42$ & $<1.32$ \\
\hline 220947 & 5500 & 3.80 & $<1.44$ & $<1.40$ \\
\hline 224701 & 5500 & 3.90 & $<1.42$ & $<1.38$ \\
\hline 224921 & 5500 & 3.90 & $<1.33$ & $<1.28$ \\
\hline 227902 & 5750 & 4.10 & $<1.69$ & $<1.67$ \\
\hline 234254 & 5350 & 3.90 & $<1.41$ & $<1.34$ \\
\hline 235569 & 5650 & 4.00 & $<1.53$ & $<1.49$ \\
\hline 243327 & 5300 & 3.70 & $<1.17$ & $<1.10$ \\
\hline 247798 & 5500 & 4.00 & $<1.34$ & $<1.29$ \\
\hline 248814 & 5500 & 4.00 & $<1.36$ & $<1.31$ \\
\hline
\end{tabular}

upper limits correspond to the lithium abundance assuming an equivalent width of $2 \sigma_{E W}$, where $\sigma_{E W}$ was computed from the $S / N$ ratio of the spectrum derived from the Cayrel (1988) formula. The choice of $2 \sigma$ implies that the abundance of each line has a probability of 0.0455 to be larger than the upper limit, if noise prevented us to detect it. For this to happen 18 times the probability is $0.0455^{18} \simeq 7 \times 10^{-25}$.

Placing a firm constraint on the lithium content of SGBa stars has some importance when related to the elusive helium abundance of this population. As will be discussed in detail in Sect. 4.6, the RGB-a/SGB-a population should possess a high helium abundance (Norris 2004; Piotto et al. 2005; Renzini 2008). All the H-burning processes, where He is produced, happen at temperatures where $\mathrm{Li}$ is destroyed. Therefore, He-rich stars should have a very low lithium content.

What is important here is that we have no detection (as was the case in Monaco et al. 2010, as well), with upper limits in the range $A(\mathrm{Li})=1.0$ to 1.7 , depending on $T_{\text {eff }}$. In subgiants with these temperatures $\mathrm{Li}$ is expected to be slightly depleted with respect to the Spite plateau (Ryan \& Deliyannis 1998; Mucciarelli et al. 2011), but still detectable precisely in the above abundance range, with a well defined "Li-ridge" (see Fig. 4 of Ryan \& Deliyannis 1998). Our upper limits suggest that the SGB-a Li abundance is below the standard "Li-ridge". Thus the absence of any Li detection in our sample of SGB-a stars provides indirect support to the notion that these stars are indeed He-rich.

Deeper observations of the hottest stars of the sample would be desirable to see if any $\mathrm{Li}$ is at all detectable. A measured $\mathrm{Li}$ abundance would provide a very strong constraint on the amount of Li-free material to be mixed with Li-normal material at the time of the star formation. In turn this would provide the necessary $\mathrm{He}$ abundance of the He-rich material in order to obtain the total He abundance implied by the CMD and abundance information of the main sequences. This would place strong constraints on the stars responsible for the nuclear processing. In the chemical evolution model of Romano et al. (2010), He is provided mostly by massive AGB stars $\left(4-5 M_{\odot}\right)$, and the Herich stars are formed from almost pure AGB ejecta. This material would certainly be Li-free, thus a detection of $\mathrm{Li}$ in the He-rich sub-populations, even at a low level, could rule out this scenario.

\section{3. $\alpha$-elements}

There were half a dozen measurable Ca lines in our spectra, complemented by one single Si line and by $3-4 \mathrm{Ti}$ I and Ti II lines, that we averaged together to produce $[\mathrm{Ti} / \mathrm{Fe}]$. Calcium and titanium appear to agree with previous literature estimates, being $\langle[\mathrm{Ca} / \mathrm{Fe}]\rangle=+0.26 \pm 0.16 \mathrm{dex}$, and $\langle[\mathrm{Ti} / \mathrm{Fe}]\rangle=+0.45 \pm 0.12$ dex . As discussed in Sect. 1, while the 0.2-0.3 dex decrease in $\alpha$ enhancement initially found by Pancino et al. (2002) and Origlia et al. (2003) is not confirmed by more recent studies quantitatively, nevertheless a decrease of 0.1 dex approximately can be seen in all sufficiently sampled studies (Norris \& Da Costa 1995; Smith 2004; Pancino 2004; Johnson \& Pilachowski 2010), and is indeed present in our data.

The lone $\mathrm{Si}$ line in our spectral range, at $6721.85 \AA$, has a theoretically computed $\log g f$ (Kurucz 1973), rather than measured in laboratory. In the abundance analysis of the Sun performed by Pancino et al. (2010) with the same models, the same code, and the same initial linelist and $\log g f$ system, this line gave an overestimated $\mathrm{Si}$ abundance by 0.19 dex. Therefore, the $[\mathrm{Si} / \mathrm{Fe}]$ values of Table 3 have been lowered by that amount in Fig. 4, and they appear to agree with the Johnson \& Pilachowski (2010) data, within the uncertainties.

Our titanium ratios also follow the trend in the Johnson \& Pilachowski (2010) data, which have a tendency to rise with $[\mathrm{Fe} / \mathrm{H}]$ as found also by other authors (Norris \& Da Costa 1995; Smith et al. 2000, to name a few). The large uncertainties on our $\mathrm{Ti}$ measurements are due to the low number of Ti I and Ti II lines ( $\simeq 4-5$, depending on the star) available in the studied spectral range; these lines are small $(\simeq 20-30 \mathrm{~m} \AA)$ and with large $E W$ errors $(\simeq 10-20 \%)$; the VALD $\log g f$ values are identical to the NIST ones, that are classified as D and thus not very accurate; as a result the abundances of the 4-5 line are largely inconsistent, and their scatter is the main reason for the the huge errobars appearing in Fig. 4. Therefore, even if a few stars appear to have $[\mathrm{Ti} / \mathrm{Fe}] \simeq 0 \mathrm{dex}$, we consider this more likely a measurement problem than an intrinsic property of these stars.

\subsection{Heavy elements}

We could measure barium by means of spectral synthesis of the $6496 \AA$ line, which was generally around $100 \mathrm{~m} \AA$. We used MOOG $^{17}$ (Sneden 1973) for spectral synthesis, in combination with the same models and atmospheric parameters used for the $E W$ abundance analysis ${ }^{18}$. We found $\langle[\mathrm{Ba} / \mathrm{Fe}]\rangle=+0.87 \pm$ 0.23 dex for the SGB-a population. Our results agree with most past studies of RGB-a stars, which found a $[\mathrm{Ba} / \mathrm{Fe}] \sim+1.0 \mathrm{dex}$ for stars with $[\mathrm{Fe} / \mathrm{H}]>-1.0$ dex (see Norris \& Da Costa 1995; Smith et al. 2000; Vanture et al. 2002, to name a few), in continuity with the MInt populations lying around $-1.5<$ $[\mathrm{Fe} / \mathrm{H}]<-1.0$ dex. NLTE corrections for our $[\mathrm{Ba} / \mathrm{Fe}]$ measurements should be lower than 0.1 dex (Korotin et al. 2011). The only study of SGB-a stars providing some barium abundance was that of Villanova et al. (2007), which appears to follows the general trend of red giants studies, having $[\mathrm{Ba} / \mathrm{Fe}] \sim+1.0 \mathrm{dex}$,

\footnotetext{
17 http://verdi.as.utexas.edu/moog.html

18 Two stars were re-analyzed with MOOG (WFI 213129 and 215700), using the same atmospheric models, linelist and atomic data, and solar reference abundances. We found an $[\mathrm{Fe} / \mathrm{H}]$ abundance ratio of -0.48 dex ( 0.03 dex lower than in Table 3) for WFI 213129 and -0.94 (0.02 dex higher) for WFI 215700, so we concluded that the two abundance calculation codes give fully compatible results.
} 
although their $[\mathrm{Fe} / \mathrm{H}]$ ratios are different from the ones derived here (see Sects. 3.5 and 4.1).

\subsection{Anti-correlations}

The only representative of the proton capture elements in our spectral range is $\mathrm{Al}$, for which we measured the $6696,6698 \AA$ doublet. We did not apply NLTE corrections to our abundances, because the used doublet should be relatively free from NLTE effects (Gehren et al. 2004; Andrievsky et al. 2008). It is interesting to recall here that both Johnson \& Pilachowski (2010) and Marino et al. (2011) found a tendency, for stars richer than $[\mathrm{Fe} / \mathrm{H}] \simeq-1.0 \mathrm{dex}$, to exhibit no (anti-)correlation among the usual elements ( $\mathrm{Na}, \mathrm{Al}, \mathrm{Mg}, \mathrm{C}, \mathrm{N}$, and $\mathrm{O}$ ). Only one homogeneous group of stars is present in the Marino et al. (2011) data, which appears roughly solar in oxygen, but highly enriched in $\mathrm{Na}([\mathrm{Na} / \mathrm{Fe}] \simeq+1 \mathrm{dex})$. The RGB-a appears also enriched in $\mathrm{Al}$ $([\mathrm{Al} / \mathrm{Fe}] \simeq+0.4 \mathrm{dex})$ in the Johnson \& Pilachowski (2010) data. Here we find a homogeneous $\mathrm{Al}$ abundance among our 18 SGBa stars, with $\langle[\mathrm{Al} / \mathrm{Fe}]\rangle=+0.32 \pm 0.14 \mathrm{dex}$, well compatible with the result of Johnson \& Pilachowski (2010) and - indirectly with Marino et al. (2011) as well, with a spread comparable to the measurement errors. We stress that the importance of this result lies not in the exact average value of $[\mathrm{Al} / \mathrm{Fe}]$ found, but in the fact that it is homogeneous among our SGB-a targets. Our result supports the finding that no (anti)-correlation appears to be present among these metal-rich stars.

\subsection{Helium overabundance}

It has been suggested by several authors (starting with Norris 2004; Piotto et al. 2005; Renzini 2008), that at least part of the MInt population and the whole RGB-a population should be enriched in helium, with a typical $Y=0.35-0.40$, depending on the author. Such a helium enriched atmosphere would be different from the usual atmospheric models employed in abundance analysis calculations (Böhm-Vitense 1979). A first order of magnitude evaluation of the impact of helium can be obtained along the lines of Paper $\mathrm{I}^{19}$, where we used the Gray (2008) formula, and an exemplificative value of $Y=0.35$, corresponding to a $A(\mathrm{He}) \simeq 0.15$ instead of 0.10 :

$$
\frac{\Delta g}{g}=\frac{4 \Delta A(\mathrm{He})}{1+4 A(\mathrm{He})} \text {. }
$$

We estimate that this would correspond to an increase - for our targets - from $\log g \simeq 4.0$ dex to 4.2 dex. This would increase our abundances by approximately $0.04 \mathrm{dex}$ in $[\mathrm{Fe} / \mathrm{H}]$, which is negligible, given the uncertainties involved in the present analysis.

To investigate the matter further, we computed helium enhanced atmosphere models for three of our targets: one warm star (WFI 227902), one cool star (WFI 216031), and one average star (WFI 220947). We kept the atmospheric parameters listed in Table 1 fixed. We used the ATLAS $12 \operatorname{code}^{20}$

\footnotetext{
${ }_{19}$ In Paper I, we used the same formula, but we made a trivial error in the computation. We estimated that, if the MInt star WFI 512115 was enriched in helium $(Y \simeq 0.35)$, we would have underestimated its $[\mathrm{Fe} / \mathrm{H}]$ by 0.08 dex because we used a $Y \simeq 0.25$ atmospheric model. However, the correct value is much smaller: 0.03 dex. Such an underestimate would be entirely within the measurement uncertainties.

20 ATLAS 12 is the only program publicly available to compute atmosphere models of arbitrary chemical composition, so it was our only choice even if the rest of our abundance analysis was made with
}

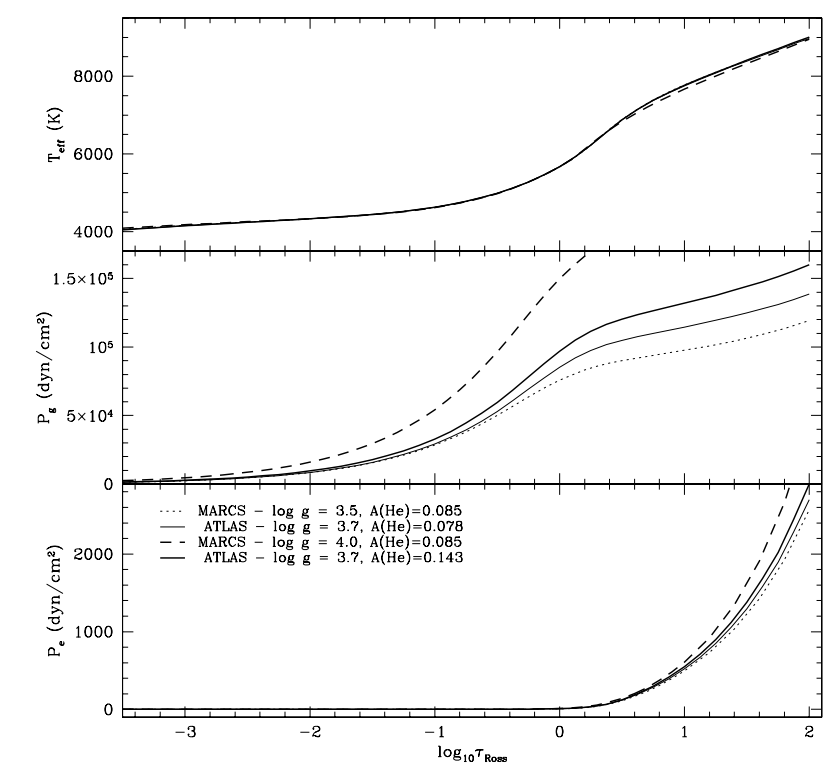

Fig. 5. Comparison of thermal and pressure structure of $5250 \mathrm{~K}$ MARCS models with specially computed ATLAS 12 models. The top panel shows the temperature variation with the logarithmic Rosseland opacity, the middle panel the gas pressure variation, and the bottom panel the electron pressure variation. The MARCS models have normal helium abundance and different gravities $(\log g=3.5$ dex plotted as a dotted line and $\log g=4.0$ dex as a dashed line). The ATLAS 12 models have normal helium (thin continuous line) and enhanced helium (thick continuous line) at a fixed gravity of 3.7 dex.

(Kurucz 2005; Castelli 2005) to compute models with a metallicity of -1.0 dex, $\alpha$-enhanced, and the resulting models for star WFI $216031\left(T_{\text {eff }}=5250 \mathrm{~K}, \log g=3.7 \mathrm{dex}\right)$ are compared with non-interpolated MARCS models ( $\log g=3.5$ and $4.0 \mathrm{dex})$ in Fig. 5. As expected, the effect of helium is similar to (but smaller than) the effect caused by an increase in gravity: the thermal structure appears almost unaffected, while the pressure structures are altered, with both gas and electron pressure increasing with helium abundance and with gravity.

We then used the computed ATLAS 12 atmospheres to recompute our abundances for the three chosen stars. We left the rest of the abundance analysis ingredients untouched: we used the same atmospheric parameters, the same EWs and linelist, the same atomic data, solar composition, and abundance calculation code. First we used the helium-normal ATLAS 12 atmospheres to compare with the MARCS model atmospheres analysis, then we compared the ATLAS 12 analysis with helium-normal and enhanced atmospheric models. The results are presented in Table 6, where it can be appreciated that both the abundance ratio difference between the MARCS and ATLAS 12 analysis, and between the ATLAS 12 analysis with normal and enhanced helium, are negligible compared to the involved uncertainties, and slightly smaller than our initial rough estimate of 0.04 dex in $[\mathrm{Fe} / \mathrm{H}]^{21}$.

MARCS models. In general differences between MARCS and ATLAS models are negligible, once the appropriate mixing-length parameters are chosen, taking into account the different formulation of the mixing-length theory in the two codes (see e.g., Bonifacio et al. 2009, Appendix A1).

21 Similarly small differences (smaller than \pm 0.03 dex) were found between He-normal and enhanced ATLAS 12 models using the Kurucz abundance calculation code instead of the Spite one, when keeping all the remaining ingredience of the calculation fixed. 
Table 6. Abundance analysis with varying helium.

\begin{tabular}{|c|c|c|c|c|}
\hline WFI 216031 & $\begin{array}{c}\text { ATLAS } \\
A(\mathrm{He})=0.085\end{array}$ & $\Delta_{\text {model }}{ }^{a}$ & $\begin{array}{c}\text { ATLAS } \\
A(\mathrm{He})=0.143\end{array}$ & $\Delta_{\text {helium }}^{b}$ \\
\hline$[\mathrm{Fe} / \mathrm{H}]$ & -0.73 & +0.02 & -0.74 & -0.01 \\
\hline$[\mathrm{Al} / \mathrm{Fe}]$ & +0.33 & -0.01 & +0.34 & +0.01 \\
\hline$[\mathrm{Ca} / \mathrm{Fe}]$ & +0.44 & -0.01 & +0.43 & -0.01 \\
\hline$[\mathrm{Ni} / \mathrm{Fe}]$ & +0.10 & 0.00 & +0.12 & +0.02 \\
\hline$[\mathrm{Si} / \mathrm{Fe}]$ & +0.85 & +0.01 & +0.87 & +0.02 \\
\hline$[\mathrm{Ti} / \mathrm{Fe}]$ & -0.02 & +0.01 & +0.01 & -0.01 \\
\hline WFI 220947 & $\begin{array}{c}\text { ATLAS } \\
A(\mathrm{He})=0.085\end{array}$ & $\Delta_{\text {model }}{ }^{a}$ & $\begin{array}{c}\text { ATLAS } \\
A(\mathrm{He})=0.143\end{array}$ & $\Delta_{\text {helium }}^{b}$ \\
\hline$[\mathrm{Fe} / \mathrm{H}]$ & -0.64 & -0.01 & -0.65 & -0.01 \\
\hline$[\mathrm{Al} / \mathrm{Fe}]$ & +0.20 & +0.01 & +0.21 & +0.01 \\
\hline$[\mathrm{Ca} / \mathrm{Fe}]$ & +0.23 & 0.00 & +0.23 & +0.00 \\
\hline$[\mathrm{Ni} / \mathrm{Fe}]$ & +0.23 & +0.01 & +0.25 & +0.02 \\
\hline$[\mathrm{Si} / \mathrm{Fe}]$ & +0.33 & 0.00 & +0.35 & +0.02 \\
\hline$[\mathrm{Ti} / \mathrm{Fe}]$ & +0.16 & +0.03 & +0.13 & -0.03 \\
\hline WFI 227902 & $\begin{array}{c}\text { ATLAS } \\
A(\mathrm{He})=0.085\end{array}$ & $\Delta_{\text {model }}{ }^{a}$ & $\begin{array}{c}\text { ATLAS } \\
A(\mathrm{He})=0.143\end{array}$ & $\Delta_{\text {helium }}^{b}$ \\
\hline$[\mathrm{Fe} / \mathrm{H}]$ & -0.66 & -0.01 & -0.65 & +0.01 \\
\hline$[\mathrm{Al} / \mathrm{Fe}]$ & +0.47 & 0.00 & +0.46 & -0.01 \\
\hline$[\mathrm{Ca} / \mathrm{Fe}]$ & +0.03 & +0.01 & -0.36 & -0.01 \\
\hline$[\mathrm{Ni} / \mathrm{Fe}]$ & -0.27 & 0.00 & -0.28 & -0.01 \\
\hline$[\mathrm{Si} / \mathrm{Fe}]$ & +0.58 & 0.00 & +0.58 & +0.00 \\
\hline$[\mathrm{Ti} / \mathrm{Fe}]$ & +0.65 & +0.02 & +0.66 & +0.01 \\
\hline
\end{tabular}

Notes. ${ }^{(a)}$ Abundance ratio difference in the sense ATLAS 12 minus MARCS. ${ }^{(b)}$ Abundance ratio difference of ATLAS 12 models in the sense of enhanced helium minus normal helium.

However, we did the above analysis by keeping the atmospheric parameters fixed. We should have probably used a different set of temperatures and gravities. For example, in the case of temperature, we used the $\mathrm{H}_{\alpha}$ line wings profile fit with a normal helium model atmosphere. If the atmosphere was enriched in helium, we can again estimate an error of approximately 0.2 dex in the synthetic spectrum gravity (an underestimate), which leads to an overestimate of the temperature of less than $50 \mathrm{~K}$ for stars of the kind analysed here. According to our calculations (see Table 4) this leads again to a negligible abundance error of the same order of magnitude of those in Table 6.

We can conclude that for the stars analysed here and in Paper I, the effect of using the wrong helium content in the atmospheric models produces errors that are negligible compared to the uncertainties involved in the abundance analysis.

\section{Summary and conclusions}

We analyzed spectra $(R \simeq 17000, S / N \simeq 25-50)$ of 18 members of $\omega$ Cen, lying on the SGB-a branch. We found that $\langle[\mathrm{Fe} / \mathrm{H}]\rangle=$ $-0.72 \pm 0.14$ dex, similarly to all past high-resolution studies of the RGB-a population (Pancino et al. 2002; Pancino 2003; Johnson \& Pilachowski 2010; Marino et al. 2011). The RGB-a is clearly the bright-end continuation of the SGB-a according to all recent high-quality photometries (Ferraro et al. 2004; Bedin et al. 2004; Villanova et al. 2007; Bellini et al. 2010). We find some disagreement only with the low-resolution spectroscopic study by Villanova et al. (2007), and we ascribe that to the lower resolution of their spectra, which cover a bluer range where line blanketing makes the continuum positioning quite difficult, and overblanketing issues might lead to abundance underestimates (Kurucz 1992; Munari et al. 2005; Bertone et al. 2008). A similar low resolution study (Sollima et al. 2005b), based on the infrared calcium triplet, gives an SGB-a abundance in good agreement with our estimate.

Abundance ratios of $\alpha$-elements were computed, finding $\langle[\alpha / \mathrm{Fe}]\rangle=+0.40 \pm 0.16$ dex. This leaves little room for a significant decrease of the $\alpha$-enhancement $(>0.2$ dex) of the $R G B-a / S G B-a$ population with respect to the more metal-poor ones. A decrease of $\Delta[\alpha / \mathrm{Fe}] \simeq 0.2$ and 0.3 dex was found by Pancino et al. (2002) and Origlia et al. (2003) respectively, but according to Pancino (2004) and Johnson \& Pilachowski (2010), this decrease should be lower, of 0.1-0.15 dex at most. While type Ia supernovae were invoked in the past to explain this decrease, a more complex situation appears from these larger samples of stars, where the $\alpha$-enhancement increases slowly with $[\mathrm{Fe} / \mathrm{H}]$, reaches its maximum in the MInt population, and then slightly decreases again in the RGB-a population. A straightforward interpretation of this behaviour probably requires detailed chemical evolution modelling. While the metal-rich end could still be explained by type Ia supernovae intervention, the lower $[\alpha / \mathrm{Fe}]$ of the metal-poor stars around $[\mathrm{Fe} / \mathrm{H}]=-1.7$ dex requires a variation in the star formation rate during the chemical enrichment history of $\omega$ Cen.

We could also measure $\mathrm{Al}$ which - together with $\mathrm{C}, \mathrm{N}, \mathrm{O}$, $\mathrm{Na}$, and $\mathrm{Mg}$ - is one of the elements that (anti)-correlate in nearly all GC studied up to now (see Gratton et al. 2004; Carretta et al. 2009, and references therein). It is interesting to note that we found a fairly homogeneous Al abundance among our SGBa targets, in good agreement with what found by Johnson $\mathcal{E}$ Pilachowski (2010) and - indirectly - by Marino et al. (2011) with their $O$ and $N a$ measurements. We measured $\langle[\mathrm{Al} / \mathrm{Fe}]\rangle=$ $+0.32 \pm 0.14 \mathrm{dex}$, where the spread is compatible with measurement errors only. The importance of this result does not lie in the exact value of the average $[\mathrm{Al} / \mathrm{Fe}]$, but in the fact that it is homogenous among our 18 SGB-a stars. The field populations of dwarf galaxies and of the Milky Way are substantially free from (anti)-correlations (Martell \& Grebel 2010), even when they are found among their GC (Letarte et al. 2006; Mucciarelli et al. 2009). Similarly, M 54 shows a clear $\mathrm{Na}-\mathrm{O}$ anti-correlation while the field population of the Sagittarius dwarf galaxy is free of it (see e.g., Monaco et al. 2005; Sbordone et al. 2007; Carretta et al. $2010)^{22}$.

In the popular scenario of the formation of $\omega$ Cen as a disrupted dwarf galaxy (see, e.g., Bekki \& Freeman 2003, and references therein), it can be speculated that, if any of the subpopulations of $\omega$ Cen is free from (anti)-correlations, that population is a likely candidate for its putative parent galaxy field population. This was discussed for the case of the VMP (very metal-poor) population defined in Paper I, and was also discussed by Pancino (2003) and Carretta et al. (2010). We have then two candidate populations for the parent galaxy field: the VMP and the RGB-a/SGB-a. However, (anti)-correlations are not completely ruled out in the case of the VMP (see Paper I; Johnson \& Pilachowski 2010; Marino et al. 2011), and they could simply be of a lesser extent. Moreover, the metallicity of the Sagittarius field population around M54 is metalrich (about -0.5 dex, Carretta et al. 2010), analogously to the SGB-a/RGB-a population in $\omega$ Cen, so the RGB-a/SGB-a should be the best candidate. It must be noted, however, that the analogy with Sagittarius and other dwarf galaxies breaks when the $\alpha$-elements are considered, being higher in $\omega$ Cen than in any

\footnotetext{
22 The case of Terzan 5 (Ferraro et al. 2009; Origlia et al. 2011) has no similarity to what presented here, because none of its two populations shows any anti-correlation and therefore this object must have had an entirely different chemical evolution.
} 
other dwarf. Also, the high [Na/Fe] of the RGB-a/SGB-a population and its narrow metallicity range are not easily explained in this scenario. Thus more work is required to assess if $\omega$ Cen is really the remnant of an accreted and disrupted dwarf galaxy, and to find its elusive relics in the field population of the Galaxy (see, e.g., Meza et al. 2005; Da Costa \& Coleman 2008; Sollima et al. 2009).

Finally, it has been speculated (see, e.g., Norris 2004; Piotto et al. 2005; Renzini 2008) that the RGB-a population should be rich in helium (with $Y \simeq 0.35-0.40$ ), and therefore we tested whether an usual abundance analysis, based on model atmospheres with a normal ( $Y \simeq 0.25$ ) content, could significantly affect the resulting abundance ratios. We calculated heliumenhanced models with ATLAS 12 and recomputed our abundances, and the results is that the effect of the helium content of the model atmospheres has a negligible impact on the resulting abundance ratios for stars of the type studied here and in Paper I. We also determined upper limits to the lithium content and found no $6708 \AA \mathrm{Li}$ I line detection in our 18 stars. This suggests that lithium in SGB-a stars is less abundant than what is typical (Ryan $\mathcal{E}$ Deliyannis 1998), and therefore lends support to the notion that these stars have enhanced helium content, as discussed in detail in Sect. 4.2.

Acknowledgements. We warmly thank M. Bellazzini and F. R. Ferraro for their advice. E.P. would like to acknowledge the hospitality of the Universidad de Concepción, Chile, where part of this work was carried out. We also thank an anonymous referee for her/his work on this paper.

\section{References}

Alonso, A., Arribas, S., \& Martínez-Roger, C. 1999, A\&AS, 140, 261 Andersen, J. 1999, Transactions of the IAU, Vol. XXIII B (Dordrecht: Kluwer) Andrievsky, S. M., Spite, M., Korotin, S. A., Spite, F., et al. 2008, A\&A, 481, 481

Barklem, P. S., Piskunov, N., \& O’Mara, B. J. 2000, A\&A, 363, 1091

Bedin, L. R., Piotto, G., Anderson, J., et al.2004, ApJ, 605, L125

Bekki, K., \& Freeman, K. C. 2003, MNRAS, 346, L11

Bellazzini, M., Ferraro, F. R., Sollima, A., Pancino, E., \& Origlia, L. 2004, A\&A, 424, 199

Bellini, A., Piotto, G., Bedin, L. R., et al. 2009, A\&A, 493, 959

Bellini, A., Bedin, L. R., Piotto, G., et al. 2010, AJ, 140, 631

Bergbusch, P. A., VandenBerg, D. A. 2001, ApJ, 556, 322

Bertone, E., Buzzoni, A., Chávez, M., \& Rodríguez-Merino, L. H. 2008, A\&A, 485,823

Bessell, M. S. 1979, PASP, 91, 589

Böhm-Vitense, E. 1979, ApJ, 234, 521

Bonifacio, P., Spite, M., Cayrel, R., et al. 2009, A\&A, 501, 519

Busso, M., Gallino, R., \& Wasserburg, G. J. 1999, ARA\&A, 37, 239

Carretta, E., Bragaglia, A., Gratton, R., \& Lucatello, S. 2009, A\&A, 505, 139

Carretta, E., Bragaglia, A., Gratton, R. G., et al. 2010, ApJ, 714, L7

Castelli, F. 2005, Mem. Soc. Astron. Ital. Supp., 8, 25

Cayrel, R. 1988, The Impact of Very High S/N Spectroscopy on Stellar Physics, 132,345

Cayrel, R., Depagne, E., Spite, M., et al. 2004, A\&A, 416, 1117

Da Costa, G. S., \& Coleman, M. G. 2008, AJ, 136, 506

Dean, J. F., Warren, P. R., \& Cousins, A. W. J. 1978, MNRAS, 183, 569

Dupree, A. K., Strader, J., \& Smith, G. H. 2011, ApJ, 728, 155

Ferraro, F. R., Sollima, A., Pancino, E., et al. 2004, ApJ, 603, L81

Ferraro, F. R., Dalessandro, E., Mucciarelli, A., et al. 2009, Nature, 462, 483

Freytag, B., Steffen, M., \& Dorch, B. 2002, Astron. Nachr., 323, 213

Freytag, B., Steffen, M., Wedemeyer-Böhm, S., \& Ludwig, H.-G. 2010, CO5BOLD User Manual, http://www.astro.uu.se/ bf/co5bold_ main.html
Gehren, T., Liang, Y. C., Shi, J. R., Zhang, H. W., \& Zhao, G. 2004, A\&A, 413, 1045

Gratton, R., Sneden, C., \& Carretta, E. 2004, ARA\&A, 42, 385

Grevesse, N., Noels, A., \& Sauval, A. J. 1996, Cosmic Abundances, 99, 117 Observatory

Grevesse, N., \& Sauval, A. J. 1998, Space Sci. Rev., 85, 161

Gray, D. F. 2008, The Observation and Analysis of Stellar Photospheres, ed. D. F. Gray (Cambridge: Cambridge University Press)

Hilker, M., \& Richtler, T. 2000, A\&A, 362, 895

Hilker, M., Kayser, A., Richtler, T., \& Willemsen, P. 2004, A\&A, 422, L9

Hughes, J., \& Wallerstein, G. 2000, AJ, 119, 1225

Hughes, J., Wallerstein, G., van Leeuwen, F., \& Hilker, M. 2004, AJ, 127, 980

Johnson, C. I., Pilachowski, C. A., Michael Rich, R., \& Fulbright, J. P. 2009, ApJ, 698, 2048

Johnson, C. I., \& Pilachowski, C. A. 2010, ApJ, 722, 1373

Korotin, S., Mishenina, T., Gorbaneva, T., \& Soubiran, C. 2011, MNRAS, 415 2093

Kupka, F., Piskunov, N., Ryabchikova, T. A., Stempels, H. C., \& Weiss, W. W. 1999, A\&AS, 138, 119

Kurucz, R. L. 1973, SAO Special Report, 351,

Kurucz, R. L. 1992, Rev. Mexicana Astron. Astrofis., 23, 181

Kurucz, R. L. 2005, Mem. Soc. Astron. Ital. Supp., 8, 14

Letarte, B., Hill, V., Jablonka, P., et al. 2006, A\&A, 453, 547

Lub, J. 2002, Omega Centauri, A Unique Window into Astrophysics, ASP Conf. Ser., 265, 95

Marino, A. F., Villanova, S., Piotto, G., et al. 2008, A\&A, 490, 625

Marino, A. F., Milone, A. P., Piotto, G., et al. 2011, ApJ, 731, 64

Martell, S. L., \& Grebel, E. K. 2010, A\&A, 519, A14

Meylan, G., Mayor, M., Duquennoy, A., \& Dubath, P. 1995, A\&A, 303, 761

Meza, A., Navarro, J. F., Abadi, M. G., \& Steinmetz, M. 2005, MNRAS, 359, 93

Monaco, L., Bellazzini, M., Bonifacio, P., et al. 2005, A\&A, 441, 141

Monaco, L., Bonifacio, P., Sbordone, L., Villanova, S., \& Pancino, E. 2010, A\&A, 519, L3

Mucciarelli, A., Origlia, L., Ferraro, F. R., \& Pancino, E. 2009, ApJ, 695, L134

Mucciarelli, A., Salaris, M., Lovisi, L., et al. 2011, MNRAS, 412, 81

Munari, U., Sordo, R., Castelli, F., \& Zwitter, T. 2005, A\&A, 442, 1127

Norris, J. E., \& Da Costa, G. S. 1995, ApJ, 447, 680

Norris, J. E. 2004, ApJ, 612, L25

Origlia, L., Ferraro, F. R., Bellazzini, M., \& Pancino, E. 2003, ApJ, 591, 916

Origlia, L., Rich, R. M., Ferraro, F. R., et al. 2011, ApJ, 726, L20

Pancino, E., Ferraro, F. R., Bellazzini, M., Piotto, G., \& Zoccali, M. 2000, ApJ, 534, L83

Pancino, E., Pasquini, L., Hill, V., Ferraro, F. R., \& Bellazzini, M. 2002, ApJ, 568, L101

Pancino, E. 2003, Ph.D. Thesis, Bologna University, Italy

Pancino, E. 2004, Origin and Evolution of the Elements [arXiv: astro-ph/0305449]

Pancino, E., Galfo, A., Ferraro, F. R., \& Bellazzini, M. 2007, ApJ, 661, L155

Pancino, E., Carrera, R., Rossetti, E., \& Gallart, C. 2010, A\&A, 511, A56

Pancino, E., Mucciarelli, A., Sbordone, L., et al. 2011, A\&A, 527, A18 (Paper I)

Piotto, G., Villanova, S., Bedin, L. R., et al. 2005, ApJ, 621, 777

Renzini, A. 2008, MNRAS, 391, 354

Rey, S.-C., Lee, Y.-W., Ree, C. H., et al. 2004, AJ, 127, 958

Romano, D., Tosi, M., Cignoni, M., et al. 2010, MNRAS, 401, 2490

Ryan, S. G., \& Deliyannis, C. P. 1998, ApJ, 500, 398

Sbordone, L., Bonifacio, P., Buonanno, R., et al. 2007, A\&A, 465, 815

Sbordone, L., Bonifacio, P., Caffau, E., et al. 2010, A\&A, 522, A26

Smith, V. V., Suntzeff, N. B., Cunha, K., et al. 2000, AJ, 119, 1239

Smith, V. V. 2004, Origin and Evolution of the Elements, 186

Sneden, C. 1973, ApJ, 184, 839

Sollima, A., Ferraro, F. R., Pancino, E., \& Bellazzini, M. 2005a, MNRAS, 357, 265

Sollima, A., Pancino, E., Ferraro, F. R., et al. 2005b, ApJ, 634, 332

Sollima, A., Bellazzini, M., Smart, R. L., et al. 2009, MNRAS, 396, 2183

Spite, M. 1967, Ann. Astrophys., 30, 685

Stanford, L. M., Da Costa, G. S., Norris, J. E., \& Cannon, R. D. 2006, ApJ, 647, 1075

Stehlé, C., \& Hutcheon, R. 1999, A\&AS, 140, 93

Stetson, P. B., \& Pancino, E. 2008, PASP, 120, 1332

Vanture, A. D., Wallerstein, G., \& Suntzeff, N. B. 2002, ApJ, 569, 984

Villanova, S., Piotto, G., King, I. R., et al. 2007, ApJ, 663, 296 